Advanced Neutron Source, the DoE Basic Energy Sciences Advisory Committee has made preliminary recommendations to a report that will be finalised in a few months. It argues that a $5 \mathrm{MW}$ Pulsed Spallation Neutron Source (PSNS) at the Oak Ridge national lab (the DoE designated preferred ălternative site), which would be competitive with the proposed European Spallation Source, cannot be built within the 1000 MSUS ceiling set previously by DoE. So the committee recommends a MW source upgradable to $5 \mathrm{MW}$.

A proposed upgrade to $1 \mathrm{MW}$ of Argonne's Intense Pulsed Neutron Source was not recommended because it would cost much more than 100 M\$US. Meanwhile, defence and energy research programmes should collaborate on plans for a proposed 1 MW long-pulse source at the Los Alamos national lab.

The committee also recommends upgrades of the 30-year old High Flux Beam Reactor and the High Flux Isotope Reactor at the Brookhaven and Oak Ridge national labs, respectively, to provide neutron capacity while the PSNS is being built. The Los Alamos, Brookhaven and Argonne labs as well as the LawrenceBerkeley lab are presently collaborating on the PSNS design.
Switzerland above Average An evaluation of physics research in Switzerland by the Swiss Research Council has concluded that the country's physicists and astrophysicists have successfully weathered the structura transition from traditional areas to a more engineering-oriented approach without loosing the competitive edge. The amount of physics and astrophysics (P\&A) research carried out equals that undertaken by most industrial countries. For instance, 1993 spending on $\mathrm{P} \& \mathrm{~A}$ as a percentage of the GDP was about the same as for Denmark in 1990 and some $20 \%$ higher than for Sweden in 1991. However, the quality of the output can be said to be above average (a relative citation index of 2.00 in 1992 is the highest among developed countries). Specific areas (computational physics, optics, microtechnology, parts of semiconductor physics) need strengthening, taking into account contributions to international organizations. Meanwhile, the government has requested preparatory work so that the Paul Scherrer Institute can submit its Swiss Light Source synchrotron proposal to parliament for a final decision in 1997. Letters of Intent from potential users are sought [http://www1.psi.ch].

\title{
Munich Reactor Approved
}

The new FRM-II research reactor in Garching near Munich received its first partial license on 9 April, thus approving the design and allowing construction of the buildings. The budget of $720 \mathrm{MDM}$ for the facility has also been settled so construction has started. A second partial license is needed for the installation of the nuclear components, and a third for the operation of the reactor.

The first license is an important milestone and marks the end of nearly 15 year's of planning and discussions. The way is now open to realize the most powerful and modern neutron source in Germany, which, when finished in about 5-6 years, will supply scientists with experimental conditions for neutron experiments superior to those available in Berlin, Garching, Geesthacht and Julich.

FRM-II, with capacity for about 40 experimental installations and instruments, will be operated by the TU Munich as a national institution. Ten instruments are budgeted for; others can be installed by interested research groups.

The $20 \mathrm{MW}$ reactor is cooled by light water and moderated by heavy water. A relatively high ratio of neutron-to-reactor power comes from the compact size of the core and the use of highly enriched U-235. The reactor will be equipped with a vertical cold neutron source, a hot source and ten horizontal, one vertical and two inclined beam tubes. The neutron flux expected at the cold sources and at the tube inputs is 4- $6 \times 10^{14} \mathrm{n} / \mathrm{cm}^{2} \mathrm{~s}^{1}$. A large beam tube will take up to six neutron guides into an experimental area with additional laboratories. Neutron switches will allow each primary guide to be split up into three partial beams, thus creating a large number of "guide-end positions". Two neutron beams can be extracted from each of the standard beam tubes to provide two instruments per beam hole. One of the horizontal beam tubes is designed for extracting charged fusion products which can be

\section{People}

The Fritz London Memorial Award for outstanding contributions to low temperature physics, awarded every three years at the International Conference on Low Temperature Physics, has been won by M.H.W. Chan (Pennsylvania State University, PA, USA.) for innovative and precise experimental studies of phase transitions in fluids; and by E.A. Cornel and C. Wieman (National Institutes of Standards and Technology and University of Colorado, USA.) for demonstrating Bose-Einstein condensation of weakly interacting atoms.

The first Surface Structure Prize awarded by the International Conference on the Structure of Surfaces (ICSOS) has been won by J.B. Pendry for far-reaching, diverse, fundamental and practical contributions to quantitative surface structure determination.

J. Solana, the new Secretary General of NATO, trained as a physicist, having been awarded a PhD by the Universidad Autónoma de Madrid. He then became professor of solid-state physics at the Universidad Complutense de Madrid, before serving as Spain's Minister for Education and Science (1988-92) and as

\section{J. Solana}

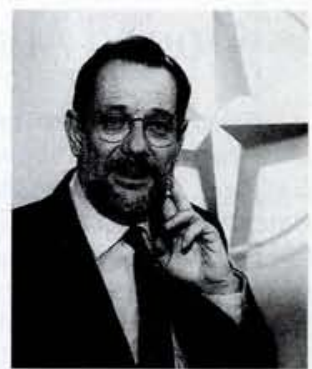

the Minister of Foreign Affairs. F. Menzinger, scientific attaché at Italy's Permanent Mission in Geneva and professor of physics at the University of Rome "Tor Vergata" succeeded R.W. Newport as the Chairman of the European Synchrotron Radiation Facility's Council on 1 January, and C. Kunz, professor of experimental physics at the University of Hamburg succeeded B. Lengeler as the Director of Research.

B. Frois $\left(\mathrm{IN}_{2} \mathrm{P}_{3}\right.$, Saclay) has been appointed chairman of IUPAP's new International Commission for Nuclear Physics. S. Gàles, the new NuPECC President, is a member.

F. Saris, formerly Director of AMOLF, Amsterdam, has become the ECN Petten's Director of Research, and G. Van Niggelkoop, Professor at the Free University, Amsterdam, has become the Director of NIKHEF, Amsterdam.

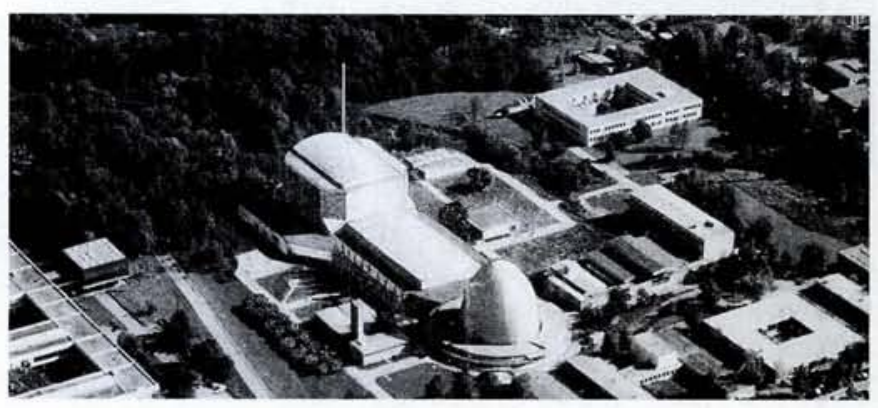

A photomontage showing a model of the new Forschungsreaktor München-II (FRM-II) behind the "egg" of the TU Munich's existing research reactor.

accelerated for heavy-ion reactions. A U-235 target at the input of another horizontal tube produces fast-fusion neutrons for cancer therapy and computer tomography with fast neutrons. Also planned is an installation for producing radioactive samples, especially phosphorus for doping semiconductor silicon.

At a time when research with neutrons becomes more important and more in demand, and with older reactors becoming more difficult to refurbish, it is natural to make the best of new possibilities. It is for this reason that we have advocated operating the reactor with highly enriched U-235. In the same spirit, we want to do our best for the equipment and the instruments. We hope that we can bring worldwide experience and knowledge to the instrumentation by having scientific visitors during the next few years. We also hope that some of the neutron beams at FRM-II will become attractive enough for scientists from elsewhere so that we can create the fruitful international atmosphere which is fundamental for research. 